\title{
„Bürgerversicherung mit mehr Hausärzten als heute"
}

\author{
Vor der Bundestagswahl präsentieren die wichtigen Parteien in der MMW ihre Gesundheitspolitik. \\ Den Abschluss machen die beiden traditionellen Volksparteien. Auf dieser Seite: Die SPD.
}

_ Grundsätzlich will die SPD, dass unser Gesundheitswesen auch angesichts des medizinischen Fortschritts und des demografischen Wandels leistungsfähig und für die Versicherten bezahlbar bleibt. Künftig sollen Arbeitgeber und Arbeitnehmer wieder zu gleichen Hälften ihren Beitrag leisten. Wir sorgen dafür, dass die Bürger sich darauf verlassen können, unabhängig von Einkommen und Wohnort die beste medizinische und pflegerische Versorgung zu bekommen.

Dafür schaffen wir eine Bürgerversicherung, in die alle einzahlen und durch die alle die notwendigen medizinischen Leistungen bekommen. Den einseitigen Zusatzbeitrag der Versicherten schaffen wir ab. Eine Zwei-Klassen-Medizin soll es nicht länger geben.

Wir wollen, dass das auch in ländlichen und strukturschwache Regionen und Stadtteilen mit sozialen Problemen eine gute und barrierefreie medizinische Versorgung Standard bleibt.

Wir wollen ein Gesundheitswesen aus einem Guss. Wir brauchen eine neue Behandlungskultur, die die Bedürfnisse der Menschen in den Mittelpunkt rückt und Zuwendung honoriert. Eine qualitativ hochwertige Versorgung kann am besten gelingen, wenn Grenzen zwischen ambulantem und stationärem Sektor überwunden werden. Dazu schaffen wir eine integrierte Bedarfsplanung der gesamten medizinischen Versorgung. Die Prävention von Krankheiten und die Rehabilitation beispielsweise

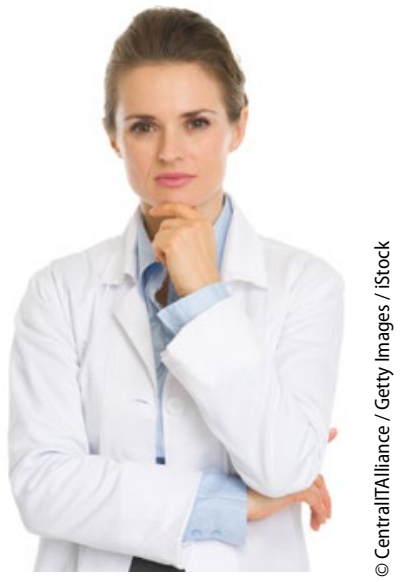

Warum sollten Ärzte

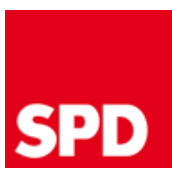

wählen?

nach einer schweren Erkrankung müssen im Rahmen dieser Planung gestärkt werden. Auch die Pflege muss einbezogen werden.

\section{Primärarztsystem bei weiterhin freier Arztwahl}

Wir brauchen darüber hinaus mehr Hausärzte als heute, denn sie sind die erste Anlaufstelle im Krankheitsfall. Die SPD steht zur freien Arztwahl. Wir sind aber auch der Auffassung, dass ein Primärarztsystem viele Vorteile bringen kann. Deshalb haben wir alle gesetzlichen Krankenkassen verpflichtet, ihren Versicherten die Mitgliedschaft in einem Hausarztvertrag anzubieten.
Die Kompetenz der Apotheker muss in die Versorgungsstrukturen effizient mit eingebunden werden. Wir wollen die Notfallversorgung verändern, um den Zugang für alle sicherzustellen. Darüber hinaus ist es unser Ziel, Menschen mit chronischen Erkrankungen von $\mathrm{Zu}$ zahlungen zu entlasten und Leistungen für Zahnersatz und Sehhilfen zu verbessern.

- Der Text wurde von der Parteizentrale der SPD erstellt

\section{Bundestagswahl: Showdown am 24. September 2017}

Vier Jahre lang ist das Land von einer Großen Koalition aus CDU/CSU und SPD regiert worden. Am 24. September 2017 werden die Karten neu gemischt. Das betrifft auch die Gesundheitspolitik. Wird die nächste Bundesregierung wieder von der Union angeführt? Wird sie wieder den Bundesgesundheitsminister stellen? Werden Parteien links von der Mitte beteiligt?

In der MMW haben die Parteien mit Regierungserfahrung über die letzten Monate ihre Ideen für das Gesundheitswesen vorgestellt. Thematisch steht ganz klar die Systemfrage im Mittelpunkt: Union und FDP setzen auf eine Fortentwicklung des Bestehenden im Kleinen, während SPD, Grüne und Linke einen Wechsel zu einer Bürgerversicherung und das Ende der privaten Krankenvollversicherung versprechen.

Es geht also für die Ärzte um Einiges. Die Wahl wird spannend.

- chy 